\title{
A serological study of canine herpesvirus-1 infection in a population of breeding bitches in Norway
}

\author{
Anette Krogenæs ${ }^{1 *}$, Vibeke Rootwelt ${ }^{2}$, Stig Larsen ${ }^{1,3}$, Lena Renström ${ }^{4}$, Wenche Farstad ${ }^{1}$ and Arve Lund ${ }^{5}$
}

\begin{abstract}
Background: Canine herpesvirus-1 (CHV1) causes a fatal hemorrhagic disease in neonatal puppies and is associated with infertility in female dogs. This study was conducted to assess the status of $\mathrm{CHV} 1$ infection in bitches in proestrus or estrus and to investigate possible risk factors by a detailed questionnaire. Blood samples were collected from healthy bitches ( $n=193$ ) not vaccinated against CHV1, aged one year or older and admitted for estrus control to the Canine Reproductive Clinical Unit, Norwegian School of Veterinary Science. The serum samples were analysed by immunoperoxidase monolayer assay and serum titers were recorded as the reciprocal value of the highest dilution producing specific cell staining.

Results: Altogether, $85.5 \%$ of the dogs had $\mathrm{CHV} 1$ titers $\geq 80$ and were classified as positive. Mean age for dogs included in the study was 4.2 years ( $95 \% \mathrm{Cl}$ 4.0-4.5), and there was no difference in age between seronegative dogs vs seropositive dogs. When grouping the seropositive dogs into three categories according to the magnitude of the titer, a total of $38.8 \%$ of the bitches displayed a weakly positive titer of $80,44.8 \%$ had moderately positive titers of 160 or 320 and $16.4 \%$ of the dogs fell into the strongly positive category with titer of $\geq 640$. No association was demonstrated when comparing CHV1 antibody titers to fertility parameters such as previous matings, pregnancies, whelpings, puppies born or condition of puppies. Further, there was no difference in seroprevalence between bitches that had been abroad for a period of time and dogs only living within a Norwegian environment. Samples from dogs collected in summer and fall displayed moderate to high antibody titers indicating recent infection with CHV1. Season, previous birth, and participation in competitions/shows explained $67-78 \%$ of the variation in antibody titer.
\end{abstract}

Conclusions: This study demonstrates that CHV1 infection is common in breeding bitches in the eastern part of Norway. Associations with putative risk factors were not identified. However, season, previous whelping, and participation in competitions/shows explained $67-78 \%$ of the variation in antibody titer.

Keywords: Canine herpesvirus, Dog, Sero-epidemiology, Reproduction

\section{Background}

There has been an increasing concern among dog breeders in Norway about canine herpesvirus-1 (CHV1) and its ability to cause reproductive problems in the bitch and perinatal puppy loss [1]. Several studies report high seroprevalence in the dog population in many European countries and there is increased movement of dogs between Norway and continental Europe.

\footnotetext{
* Correspondence: anette.krogenaes@nmbu.no

'Department of Production Animal Clinical Sciences, Faculty of Veterinary Medicine and Biosciences, Norwegian University of Life Sciences, Postboks 5003, NO-1432 Ås, Norway

Full list of author information is available at the end of the article
}

CHV1 is an alphaherpesvirus which was first reported from the USA in the early 1960s [2]. In neonatal puppies, the virus can produce a systemic fatal haemorrhagic infection causing focal necrosis in parenchymatous organs of puppies up to 2-weeks-old [3,4]. CHV1 is of low pathogenicity in puppies older than 5 weeks [5]. In the adult dog, the infection is usually asymptomatic, but may cause upper respiratory infection [3] and ocular disease [6,7]. However, in the bitch herpesvirus infection has been associated with reproductive problems, such as low conception rate, fetal resorption, abortion, stillborn or weak puppies and small litter size both in experimental $[8,9]$ and epidemiological studies [10-12]. Oronasal

\section{Biomed Central}


transmission is considered to be the main route of infection, but genital and transplacental transmission may also occur $[8,13,14]$.

In a recent study from Norway, $80 \%$ of the dogs in the general adult population were classified as seropositive [15]. No difference was observed between genders, but a significant geographical variation was demonstrated. In other countries the reported prevalence of antibodies against CHV1 varies from approximately $20 \%$ to $94 \%$ in healthy dog populations [16-18]. Some studies have reported the median antibody titer to be significantly higher in dogs from kennels with reproductive problems than in those from kennels without fertility disorders [10,19]. Different factors might influence the seroprevalence in populations such as age distribution, number of dogs in kennels and households and hygienic conditions, but the conclusions have been inconsistent $[11,12,15,19,20]$.

The aim of this study was to estimate the seroprevalence of CHV1 antibodies in reproductively active bitches and to investigate possible risk factors.

\section{Material and methods Study design, animals and sampling}

A cross-sectional study design was applied in this investigation. A total of 193 purebred bitches admitted for routine estrus control to the Canine Reproductive Clinical Unit at the Norwegian School of Veterinary Science were included in the study. All were healthy, one year of age or older (up to 9-years-old) and had not been vaccinated against herpesvirus infection. The dogs came from eastern Norway. All dog breeds were accepted for sampling and 66 different breeds are represented in this material. Of the 193 blood samples, 89 samples were collected during winter (December-February), 74 during spring (March-May), 20 during summer (June-August) and 10 during fall (September-November). In total, 57.3\% of the bitches had been pregnant earlier, whereas the remaining came for control before their first mating.

The study was conducted in agreement with the provisions enforced by the Norwegian Animal Research Authority (NARA).

The dog owners supplied information according to a questionnaire including a number of putative risk factors as last estrus, previous mating, whelping and the status of puppies. Further, information about travelling abroad, participation in dog shows, competitions and hunting trials the last year were included (see Additional file 1).

The dog owners received information on the background and purpose of the study and gave their written consent.

In the clinic, the routine procedure included ocular inspection of vulva, examination of vaginal smears to evaluate cells from vagina and progesterone analysis of serum to estimate ovulation time and optimal time for mating or artificial insemination. In addition, blood samples were taken for analysis of antibodies against CHV1. These blood samples were allowed to clot at room temperature for 1-3 hours before centrifugation at $3000 \mathrm{rpm}$ for 10 minutes. The serum fraction was carefully harvested and kept at $-20^{\circ} \mathrm{C}$ until analysis.

\section{Immunoperoxidase monolayer assay (IPMA)}

The IPMA for demonstrating antibodies to CHV1 was performed as described [15] and previously used both in epidemiological [10,15] and prospective [21] studies. IPMA is a virus neutralisation test. Briefly, monolayers of $\mathrm{CHV}$ infected Madin-Darby canine kidney cells in 96-well microtiter plates were incubated with twofold dilutions $1: 10$ to $1: 1280$ of test sera. Following washing and incubation steps, secondary peroxidase-conjugated rabbit anti-dog immunoglobulin $G$ was added (DAKO, Denmark), and subsequently the substrate 3-amino9-ethylcarbazole (Invitrogen AB, Sweden). Positive and negative serum samples were included. Serum titers were recorded as the reciprocal value of the highest dilution producing specific cell staining. Titers equal to or above 80 were considered positive for exposure to CHV1. Further, increasing antibody titers were categorized as; $80=$ weakly positive, 160 and $320=$ moderately positive, $640 \geq$ strongly positive.

The validation of the IPMA showed a sensitivity of about $90 \%$ compared to an in-house ELISA test (National Veterinary Institute, Sweden) used in a vaccine trial on sera from dogs vaccinated against CHV. Twenty-seven serum samples were collected from 13 dogs at intervals during the trial. Estimation of the specificity is not available.

\section{Statistical analyses}

Categorical variables are given in simple contingency tables with numbers and percentages. Continuously distributed variables are expressed as mean values with $95 \%$ confidence intervals. All comparisons between groups were performed two-tailed and differences considered significant at a level of $5 \%$.

Contingency Table Analysis was first performed for comparison of groups with regard to categorical variables [22]. For comparison of assumed continuously distributed variables, Analysis of Variance (ANOVA) was used [23]. The variables which in the single analysis indicated differences between positive and negative titers were included in a forward and backward stepwise logistic regression model [24]. The CHV1 titer categories were used as the dependant variables. The set of independent variables contributing to discriminate against negative titer were season, previous birth and participation in competitions. This set was included in the final logistic regression analysis. Reciever Operating Characteristics (ROC) 
analysis was used to estimate the accuracy of CHV1 titer classification. The accuracy is said to be good if the area under the ROC-curve (Auc) is larger than 0.7. An area close to 0.5 represents no difference against negative titer. The logistic model was evaluated using the HosmerLemeshow test and ROC analysis [25].

\section{Results}

No pathological conditions were found during the clinical examination and none of the dogs received any kind of medical treatment at that time. During the last year, only one dog had been treated to end a previous estrus and none of the dogs had received long-lasting cortisone treatment. Altogether $85.5 \%$ of the dogs had CHV1 antibody titers $\geq 80$ and were classified as positive. Mean age for dogs included in the study was 4.2 years $(95 \%$ CI 4.0-4.5), and there was no difference in age between the groups of seronegative dogs $v s$ seropositive dogs. Further, there were no significant differences in titer categories between dogs aged one, two and up to nine years old. No association was demonstrated when comparing CHV1 antibody titers and fertility parameters such as previous matings, pregnancies or whelpings. Furthermore, no difference was observed between negative and positive dogs with respect to information on the condition of puppies born previously (Table 1).

All of the bitches with negative titers had participated in dog shows and competitions, whereas $11.3 \%$ of the positive bitches had never attended such activities $(P=0.017)$. There was no association between antibody titer and travel abroad (Table 1).

Of all positive dogs, $38.8 \%$ displayed a titer of 80 , whereas $44.8 \%$ and $16.4 \%$ of the dogs fell into the moderately and strongly positive categories, respectively (Table 2). There was no difference in age between the different titer categories. Further, there were no significant differences between the different titer categories with respect to previous matings, pregnancies and whelpings or conditions of puppies born. However, though not significant different, 11 of 27 (40.7\%) dog owners in the highest titer category reported problems with getting the bitches pregnant after mating compared to 7 of $28(25.0 \%)$ of the negative dogs and 29 of 135 (21.5\%, 3 missing observations) in the weakly and moderately positive titer categories (Table 2). Travel abroad had no influence on titer value, whereas significantly fewer dogs within all positive titer categories had participated in dog shows, competitions or hunting trials compared to dogs with negative titers $(P=0.01$, Table 2$)$. Samples from all the negative dogs were collected during winter and spring. Of the positive dogs, $81.8 \%(\mathrm{n}=135)$ samples were collected during these two seasons. During summer and fall, only bitches from the two highest titer
Table 1 Variables within positive vs negative titer categories

\begin{tabular}{llccc}
\hline Variables & Outcome & \multicolumn{2}{c}{ Titer category } & p-values \\
\cline { 2 - 4 } & & Negative & Positive & \\
\hline Previous pregnancy & No & 10 & 72 & \\
& Yes & 18 & 92 & 0.61 \\
& Missing & 0 & 1 & \\
Previous whelping & No & 14 & 83 & \\
& Yes & 14 & 80 & 0.73 \\
Condition of puppies & Normal & 10 & 60 & \\
born previously & Weak & 0 & 1 & 0.83 \\
& Some dead & 4 & 18 & \\
& Missing & 0 & 3 & \\
Previous unsuccessful & No & 21 & 122 & \\
attempt to get pregnant & Yes & 7 & 40 & 0.62 \\
& Missing & 0 & 3 & \\
Stay abroad last year & No & 10 & 70 & \\
& Yes & 18 & 90 & 0.33 \\
& Missing & 0 & 5 & \\
Participation in & No & 0 & 18 & \\
competitions last year & Yes & 28 & 141 & 0.017 \\
& Missing & 0 & 6 & \\
\hline
\end{tabular}

categories were represented $(P<0.01$, Table 3$)$. By multivariable analysis, it was shown that each of the parameters season, previous whelping, and participation in competitions/shows contributed significantly (67-78\%) to the different antibody titer categories (Figure 1).

\section{Discussion}

This study shows that the majority of clinically healthy bitches presented to the Canine Reproductive Clinical Unit at the Norwegian School of Veterinary Science, have been infected by CHV1.

In a previous Norwegian study including both genders, we demonstrated significant geographical differences ranging between $58.5 \%$ in eastern Norway and $98 \%$ in mid-Norway [15]. When comparing the seroprevalence in the two dog populations originating from eastern Norway and collected in the same period, there is a difference of $85.5 \%$ (breeding bitches in this study) vs $58.5 \%$ (dogs of both genders in the previous study). This finding might indicate that reproductively active bitches admitted to the reproduction clinic have a greater risk of CHV1 exposure and subsequent infection compared to the general dog population in this part of the country.

All bitches in the present study were in proestrus or estrus. Although clear evidence of an association 
Table 2 Variables within the three positive vs negative titer categories

\begin{tabular}{|c|c|c|c|c|c|c|}
\hline & \multirow[t]{2}{*}{ Outcome } & \multicolumn{4}{|c|}{ Titer categories } & \multirow[t]{2}{*}{ p-values } \\
\hline & & Negative & Weakly $^{\mathrm{a}}$ positive & Moderately ${ }^{\mathbf{b}}$ positive & Strongly ${ }^{c}$ positive & \\
\hline \multirow[t]{3}{*}{ Previous pregnancy } & No & 10 & 33 & 29 & 10 & \\
\hline & Yes & 18 & 31 & 44 & 17 & 0.53 \\
\hline & Missing & 0 & 0 & 1 & 0 & \\
\hline \multirow[t]{3}{*}{ Previous whelping } & No & 14 & 37 & 33 & 13 & \\
\hline & Yes & 14 & 26 & 40 & 14 & 0.68 \\
\hline & Missing & 0 & 1 & 1 & 0 & \\
\hline \multirow[t]{4}{*}{ Condition puppies born previously } & Normal & 10 & 20 & 30 & 10 & \\
\hline & Weak & 0 & 1 & 0 & 0 & 0.78 \\
\hline & Some dead & 4 & 5 & 10 & 3 & \\
\hline & Missing & 0 & 0 & 0 & 1 & \\
\hline \multirow{3}{*}{$\begin{array}{l}\text { Previous unsuccessful attempt } \\
\text { to get pregnant }\end{array}$} & No & 21 & 51 & 55 & 16 & \\
\hline & Yes & 7 & 12 & 17 & 11 & 0.34 \\
\hline & Missing & 0 & 1 & 2 & 0 & \\
\hline \multirow[t]{3}{*}{ Stay abroad last year } & No & 10 & 28 & 30 & 12 & \\
\hline & Yes & 18 & 36 & 41 & 13 & 0.24 \\
\hline & Missing & 0 & 0 & 3 & 2 & \\
\hline \multirow[t]{3}{*}{ Participation in competitions last year } & No & 0 & 5 & 11 & 2 & \\
\hline & Yes & 28 & 59 & 59 & 23 & 0.01 \\
\hline & Missing & 0 & 0 & 4 & 2 & \\
\hline
\end{tabular}

Weakly positive $=80$.

${ }^{\mathrm{b}}$ Moderately positive $=160$ and 320 .

'Strongly positive $\geq 640$.

between circulating reproductive hormone concentrations and viral activation have not been demonstrated, Ronsse et al. [12] reported fluctuations in CHV1 antibody titers related to cycle stage. They found slightly higher titers in estrus and significantly lower titers in early di-estrus [11]. Both 17- $\beta$ estradiol and medroxyprogesterone acetate have been shown to promote herpes simplex virus type 1 reactivation in mice $[26,27]$. Evermann et al. [20] listed risk factors for reproductive disease in the bitch, and cycle stage was identified as one of 8 factors having a positive correlation with disease. Whether estrus itself is a potential stressor which can reactivate a latent infection or increase susceptibility for a new infection remains to be seen. However, clinical signs of reactivation have been described to occur more frequently during heat and around parturition [14]. Psychological stress has also been suggested to contribute to reactivation of latent herpes simplex virus in humans $[28,29]$.

In Norway, few breeding dogs are kept in kennels, so our data are not directly comparable to kenneled dogs in studies from other countries. Babaei et al. [30] and

Table 3 Canine herpesvirus-1 serological classification in the total material $(n=193)$ by season of the year

\begin{tabular}{|c|c|c|c|c|c|}
\hline \multirow[t]{2}{*}{ Titer } & Winter $^{\mathrm{a}}$ & Spring $^{\mathbf{b}}$ & Summer ${ }^{c}$ & Fall $^{d}$ & Total \\
\hline & No (\%) & No (\%) & No (\%) & No (\%) & No (\%) \\
\hline Negative & $17(8.8)$ & $11(5.7)$ & $0(0)$ & $0(0)$ & $28(14.5)$ \\
\hline Weakly positive & $37(19.2)$ & $27(14.0)$ & $0(0)$ & $0(0)$ & $64(33.2)$ \\
\hline Moderately positive & $32(16.6)$ & $25(13.0)$ & $11(5.7)$ & $6(3.1)$ & $74(38.3)$ \\
\hline Strongly positive & $3(1.6)$ & $11(5.7)$ & $9(4.7)$ & $4(2.1)$ & $27(14.0)$ \\
\hline Total & $89(46.1)$ & $74(38.3)$ & $20(10.4)$ & $10(5.2)$ & $193(100$ \\
\hline
\end{tabular}

${ }^{a}$ Winter: December-February.

bspring: March-May.

'Summer: June-August.

${ }^{\mathrm{d}}$ Fall: September-November. 


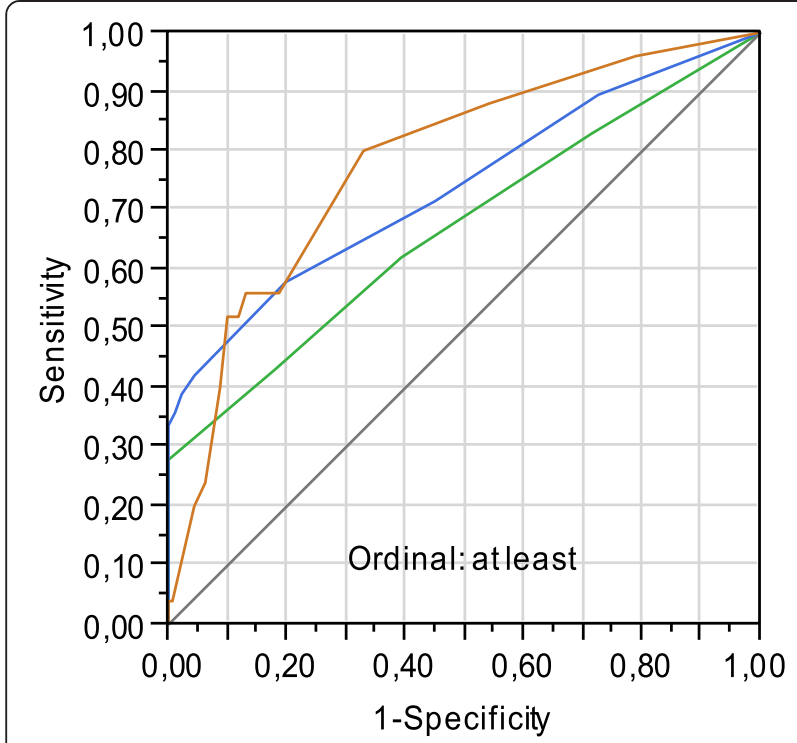

Figure 1 Receiver Operating Characteristic (ROC) including season, previous birth and competitions/shows. Weakly positive (green line) results in an area of 0.67 , moderately positive (blue line) 0.74 and strongly positive (orange line) 0.78 . The accuracy is said to be good if the area under the ROCcurve (Auc) is larger than 0.7. An area close to 0.5 represents no difference against negative titer.

Ronsse et al. [18] could not demonstrate differences in CHV1 seroprevalence between privately owned inhouse pets and kennelled dogs. Nöthling et al. [19] observed that seroprevalence was independent of kennel size, whereas Ronsse et al. [11] found higher titers in kennels with 6-30 dogs than in those with fewer dogs.

There was no difference in age between the seronegative and the seropositive bitches, and the seroprevalence did not increase with age. This is in contrast to our previous study [15] and can be explained by less variation in age in the breeding bitches in this study compared to dogs in the general population.

In the current study, there were no significant correlations between titer category and breeding status, such as previous matings, previous whelping, failing to get the bitch pregnant or conditions of puppies. This is in accordance with Ronsse et al. [18]. In the present study, the time between previous mating and sampling is variable, and the titer at previous mating is unknown. But, interestingly, owners reported increasing problems of getting the bitches pregnant after previous mating in the highest titer category compared to the others. Recent CHV1 infection coinciding with early stage of pregnancy may result in fetal loss and this could be a plausible explanation. Several authors have demonstrated an association between $\mathrm{CHV} 1$ serological status and reproductive problems. Dahlbom et al. [10] found that dogs from kennels with reproductive problems had significantly higher CHV1 titers than dogs from kennels without reproduction problems. Ronsse et al. [11] demonstrated an association between serological status and a history of abortion in bitches. Furthermore, Van Gucht et al. [31] found a relation between the presence of positive breeding bitches and neonatal death and/or infertility in the kennel.

Travel abroad with breeding dogs is quite common in Norway and is confirmed by dog-owners in this investigation for both antibody positive and -negative dogs. However, travel abroad had no influence on the titer values. This was also the fact when this was tested as a separate risk factor in our previous study of dogs in different parts of Norway [15]. By multivariable analysis, it was shown that the variable travel abroad contributed to a better classification of seropositive dogs in our previous study, which was not the case in this study with fewer dogs included. Surprisingly, significantly fewer dogs within all positive titer categories had participated in dog shows, competitions or hunting trials compared to dogs with negative titers. This might be related to the fact that the owners of the bitches in this study are committed, well-informed breeders and even though they travel or participate in different competitions, they keep their dogs under more controlled conditions than dog owners in general. The attitude and behavior of the owners in addition to the very low number of dogs not participating in competitions, might therefore be possible confounders in this study. We can conclude that the endemic status of CHV1 infection in the Norwegian dog population provides ample opportunities for spread of the virus. Staying abroad or participation in competitions/ shows does not seem to add an extra risk of contracting infection in this study.

In our data, $57.3 \%$ of the dogs had been pregnant prior to sampling. No difference in seroprevalence between this group of bitches and those being mated for the first time was demonstrated, indicating that the oronasal infection route is the most likely way of virus transmission between dogs. This finding is in accordance with other studies $[12,15,18]$ of adult dog populations. However, Babaei et al. [30], suggest that oronasal transmission of CHV1 may be epidemiologically less important than venereal transmission. In their study, no seropositive dog was detected in animals younger than 12 months of age. It might be important to emphasize that the overall CHV1 seroprevalence was estimated to $20.7 \%$, which is very low.

In spite of the high seroprevalence in reproductively active dogs, we have the opinion that fertility problems and loss of newborn puppies due to CHV1 infection at present are of minor importance in Norway. There are no epidemiological data available regarding the prevalence of reproduction disorders in bitches. However, two recent studies on postnatal puppy mortality have been 
published. Indrebø et al. [32] reported a total puppy loss to be $6.9 \%$ during the first three weeks of life in a selected population of four breeds. Etiological diagnosis was not recorded, and the authors concluded that impact of CHV1 infection was unlikely because of rare occurrence and no evidence indicating this disease. Tønnessen et al. [33] reported perinatal mortality in a large-scale observational study including 10.810 litters and 224 breeds. In total, perinatal mortality was observed in $8 \%$ of the puppies $(4.3 \%$ stillborn and $3.7 \%$ died before age of 8 days). Autopsy was not performed and causes for perinatal mortality not pursued. Obviously, there is need for follow-up investigations to find etiological causes of these clinical manifestations. At the Norwegian Veterinary Institute in Oslo, CHV1 has been diagnosed sporadically in newborn puppies (Øyvor Kolbjørnsen, personal communication). There is most likely an underdiagnosing of CHV1 infection because few puppies are submitted for autopsy due to high costs. In Norway, dogs are kept in households usually including one family dog, occasionally two, and there are few big breeding kennels [34]. Management and hygienic conditions are generally good and the stress level low probably contributing to less impact of CHV1. The fact that most bitches are seropositive to $\mathrm{CHV} 1$ following natural immunization, may protect the puppies against disease. Further, satisfactory vaccine coverage against other diseases, provide fetuses and puppies with good general protection against infection.

Interestingly, moderately positive and highly positive bitches were found only in dogs sampled during summer and fall, whereas no negative or weakly positive dogs were sampled in these seasons. This might indicate that the dogs are more exposed to new infections or reexpression of latent infections during the summer and fall, which could be due to increased outdoor activities in general and contact with other dogs in this period. Our findings may explain observations by Tønnessen et al. [33] that litters born during fall had a higher risk of experiencing early neonatal mortality than litters born during other seasons.

The three parameters of season, previous birth and participation in competitions/shows increase the probability of correct classification between the different titer categories to between 70 to $80 \%$.

Vaccination against CHV1 infection is used in some countries. In Norway, the herpesvirus vaccine is a noncore vaccine. Vaccination is not routinely recommended for breeding bitches. Nevertheless, veterinarians experience an increased demand for vaccination from owners concerned about CHV1 infection and impact of their breeding success. In case of an increased risk of CHV1 infection by recent contact with other bitches that have aborted, borne weak puppies or suffered increased neonatal mortality due to $\mathrm{CHV} 1$, vaccination should be considered in young females entering into their first or second pregnancy. In bitches with a positive CHV1 antibody titer from natural infection, protective efficacy is difficult to evaluate. We consider the status of majority of these dogs as being protected. Moreover, if reactivation of virus occurs, this is likely to boost the immune response and increase level of immunity. However, there might be relevant to consider vaccination even though naturally acquired antibodies are present, for example in older breeding bitches from about six years age. Here a reactivation may be more likely to cause problems since aging is associated with decreased immune responsiveness and increased susceptibility to infectious diseases $[20,35,36]$.

\section{Conclusions}

This study demonstrates that CHV1 infection is common in reproductively active bitches. There is no correlation between high seroprevalences and previously reported reproductive disorders in connection to matings, pregnancies, whelpings or mortality in newborn puppies in the bitches' life. Travelling abroad the last year did not influence antibody titer. All of the negative bitches, but only $81.8 \%$ of the positive bitches had participated in competitions/shows the last year, which indicates that in this data, these activities were not related to increased risk of herpesvirus infection. By multivariable analysis, it was shown that each of the parameters season, previous birth, and participation in competitions/shows explained $67-78 \%$ of the variation in antibody titer.

\section{Additional file}

Additional file 1: Questionnaire used in the study.

\section{Abbreviations}

CHV1: Canine herpesvirus-1; IPMA: Immunoperoxidase monolayer assay; ANOVA: Analysis of variance; ROC: Reciever operating characteristics; Auc: Area under the ROC-curve.

\section{Competing interests}

The authors declare that they have no competing interests.

\section{Authors' contributions}

$A K, A L, V R$ and SL participated in the discussion on the study design. AK did the clinical work, collected the samples and performed the questionnaires. $L R$ was responsible for the serum analysis. AK, AL, VR, SL and WF participated in interpretation of the data. AK, AL, VR, SL, WF and LR helped to draft the manuscript. AK wrote the final manuscript. All authors read and approved the final manuscript.

\section{Acknowledgements}

We are grateful for financial support from Dyrlege Smidts Stiftelse. We would also like to thank Eva Liljekvist for skillful laboratory work, Øyvor Kolbjørnsen for giving information about diagnosed CHV1 infections at the Norwegian Veterinary Institute in Oslo. 


\section{Author details}

'Department of Production Animal Clinical Sciences, Faculty of Veterinary Medicine and Biosciences, Norwegian University of Life Sciences, Postboks 5003, NO-1432 Ås, Norway. ${ }^{2}$ Department of Companion Animal Clinical Sciences, Faculty of Veterinary Medicine and Biosciences, Norwegian University of Life Sciences, Postboks 5003, NO-1432 Ås, Norway. ${ }^{3}$ Center of Epidemiology and Biostatistics, Faculty of Veterinary Medicine and Biosciences, Norwegian University of Life Sciences, Postboks 5003, NO-1432 Ås, Norway. ${ }^{4}$ Department of Virology, Immunobiology and Parasitology, National Veterinary Institute, Ulls Väg 2B, 75189 Uppsala, Sweden. ${ }^{5}$ Department of Health Surveillance, Norwegian Veterinary Institute, Ullevålsveien 68, Postboks 750 Sentrum, N-0106 Oslo, Norway.

Received: 3 May 2013 Accepted: 27 March 2014

Published: 2 April 2014

\section{References}

1. Rootwelt V, Lund A, Krogenæs A: Herpes virus infection in the dog - a review. Eur J Comp Anim Prac 2011, 21:1-7.

2. Carmichael LE, Squire RA, Krook L: Clinical and pathological features of a fatal viral disease of newborn pups. Am J Vet Res 1965, 26:803-814.

3. Prydie J, Harrison MJ, Graham J: Isolation of a canine herpesvirus. Vet Rec 1966, 79:660-661.

4. Carmichael LE, Greene CE: Canine herpes virus infection. In Infectious Diseases of the Dog and Cat. Edited by Greene CE. Philadelphia: WB Saunders; 1998:28-32.

5. Appel MJG, Menegus M, Parsonson IM, Carmichael LE: Pathogenesis of canine herpes virus in specific-pathogen-free dogs: 5- to 12-week-old pups. Am J Vet Res 1969, 30:2067-2073.

6. Karpas A, Garcia FG, Calvo F, Cross RE: Experimental production of canine tracheobronchitis (kennel cough) with canine herpesvirus isolated from naturally infected dogs. Am J Vet Res 1968, 29:1251-1257.

7. Ledbetter EC, Riis RC, Kern TJ, Schatzberg SJ: Corneal ulceration associated with naturally occurring canine herpesvirus-1 infection in two adult dogs. J Am Vet Med Assoc 2006, 229:376-384.

8. Hashimoto A, Hirai K, Yamaguchi T, Fujimoto Y: Experimental transplacental infection of pregnant dogs with canine herpesvirus. Am J Vet Res 1982, 43:844-850.

9. Hashimoto A, Hirai K, Suzuki Y, Fujimoto Y: Experimental transplacental transmission of canine herpesvirus in pregnant bitches during the second trimester of gestation. Am J Vet Res 1983, 44:610-614

10. Dahlbom M, Johnsson M, Myllys V, Taponen J, Andersson M: Seroprevalence of canine herpesvirus-1 and Brucella canis in Finnish breeding kennels with and without reproductive problems. Reprod Domest Anim 2009, 44:128-131.

11. Ronsse V, Verstegen J, Onclin K, Farnir F, Poulet H: Risk factors and reproductive disorders associated with canine herpesvirus-1 (CHV-1). Theriogenology 2004, 61:619-636.

12. Ronsse V, Verstegen J, Thiry E, Onclin K, Aeberlé C, Brunet S, Poulet $\mathrm{H}$ : Canine herpesvirus-1 (CHV-1): clinical, serological and virological patterns in breeding colonies. Theriogenology 2005, 64:61-74.

13. Cornwell HJC, Wright NG: Neonatal canine herpesvirus infection: a review of present knowledge. Vet Rec 1969, 84:2-6.

14. Anvik JO: Clinical considerations of canine herpesvirus infection. Vet Med 1991, 4:394-403.

15. Krogenæs A, Rootwelt V, Larsen S, Sjøberg EK, Akselsen B, Skår TM, Myhre SS, Renström LHM, Klingeborn B, Lund A: A serological study of canine herpes virus -1 infection in the adult dog population. Theriogenology 2012, 78:153-158.

16. Reading MJ, Field HJ: A serological study of canine herpes virus-1 infection in the English dog population. Arch Virol 1998, 143:1477-1488

17. Rijsewijk FAM, Luiten EJ, Daus FJ, van der Heijden RW, van Oirschot JT: Prevalence of antibodies against canine herpesvirus 1 in the dogs in The Netherlands in 1997-1998. Vet Microbiol 1999, 65:1-7.

18. Ronsse V, Verstegen J, Onclin K, Guiot AL, Aeberlé C, Nauwynck HJ, Poulet $\mathrm{H}$ : Seroprevalence of canine herpesvirus-1 in the Belgian dog population in 2000. Reprod Domest Anim 2002, 37:299-304.
19. Nöthling JO, Hüssy D, Steckler D, Ackermann M: Seroprevalence of canine herpesvirus in breeding kennels in the Gauteng Province of South Africa. Theriogenology 2008, 69:273-282.

20. Evermann JF, Ledbetter EC, Maes RK: Canine reproductive, respiratory and ocular diseases due to canine herpesvirus. Vet Clin Small Anim 2011, 41:1097-1120.

21. Ström Holst B, Hagberg Gustavsson M, Grapperon-Mathis M, Lilliehöök I, Johannisson A, Isaksson M, Lindhe A, Axnér E: Canine herpesvirus during pregnancy and non-pregnant luteal phase. Reprod Domest Anim 2012, 6:362-365.

22. Agresti A: Categorical Data Analysis. 2nd edition. New York: John Wiley and Sons, INC; 2002:36-102.

23. Altman DG: Practical Statistics for Medical Research. London: Chapman and Hall; 1991.

24. Hosmer DW, Lemeshow S: Applied Logistic Regression. 2nd edition. New York: John Wiley \& Sons, INC; 2000.

25. Zweig MH, Campbell G: Receiver-operating characteristic (ROC) plots: a fundamental evaluation tool in clinical medicine. Clin Chem 1993, 39:561-577.

26. Cherpes TL, Busch JL, Sheridan BS, Harvey SA, Hendricks RL: Medroxyprogesterone acetate inhibits CD8+ T cell viral-specific effector function and induces herpes simplex virus type 1 reactivation. $J$ Immunol 2008, 181:969-975.

27. Vicetti Miguel RD, Sheridan BS, Harvey SA, Schreineer RS, Hendricks RL, Cherpes TL: 17-beta estradiol promotion of herpes simplex virus type 1 reactivation is estrogen receptor dependant. J Virol 2010, 84:565-572.

28. Grinde B: Herpesviruses: latency and reactivation - viral strategies and host response. J Oral Microbiol 2013, 5:22766. http://www. journaloforalmicrobiology.net/index.php/jom/article/view/22766 (27.1.2014).

29. Padgett DA, Sheridan JF, Dorne J, Berntson GG, Candelora J, Glaser R: Social stress and the reactivation of latent herpes simplex virus type 1 . Proc Natl Acad Sci 1998, 95:7231-7235.

30. Babaei H, Akhtardanesh B, Ghanbarpour R, Namjoo A: Serological evidence of canine herpesvirus-1 in dogs of Kerman City, south-east of Iran. Transbound Emerg Dis 2010, 57:348-351.

31. Van Gucht S, Nauwynck H, Pensaert M: Prevalence of canine herpesvirus in kennels and the possible association with fertility problems and neonatal death. Vlaams Diergeneeskd Tijdschr 2001, 70:204-211.

32. Indrebø A, Trangerud C, Moe L: Canine neonatal mortality in four large breeds. Acta Vet Scand 2007, 49(Suppl 1):2.

33. Tønnessen R, Sverdrup Borge K, Nødtvedt A, Indrebø A: Canine perinatal mortality: a cohort study of 224 breeds. Theriogenology 2012, 77:1788-1801

34. Mejdell C: Cat or canary bird - $37 \%$ of Norwegian households have pets. Norw Vet J 2003, 115:104-105.

35. Hogenesch $\mathrm{H}$, Thompson S: Effect of ageing on the immune response of dogs to vaccines. J Comp Path 2010, 142:74-77.

36. Day M: Ageing, immunoscenescence and inflammageing in the dog and cat. J Comp Path 2010, 142:S60-S69.

doi:10.1186/1751-0147-56-19

Cite this article as: Krogenæs et al:: A serological study of canine herpesvirus-1 infection in a population of breeding bitches in Norway. Acta Veterinaria Scandinavica 2014 56:19. 